\title{
Epidemiology of tsetse flies and trypanosomes with a case study in Ethiopia'
}

\author{
Reta Duguma Abdi \\ Department of Animal Science, Institute of Agriculture, University of Tennessee \\ College of Veterinary Medicine and Agriculture, Addis Ababa University
}

\begin{abstract}
The aim of this study was to quantify the abundance of tsetse flies using traditional traps and using anti-tsetse saliva ELISA test and the trypanosome infection prevalence in the tsetse flies and cattle. An area-wide tsetse fly and cattle trypanosomosis survey was conducted in Ethiopia. Groups of mice and pigs were experimentally exposed to G. m. morsitans bite to develop total saliva and rTsal ELISA tests that are capable to detect and monitor the induced anti-saliva antibodies of tsetse following host exposure to tsetse bite. A meta-analysis was conducted on prevalence of trypanosome infection in tsetse flies in the field or in laboratory-controlled conditions. Published information available since I 950 os was reviewed. The findings indicated that tsetse flies and trypanosome infections of cattle are widely distributed in Ethiopia and their abundance is affected by spatial factors such as altitude, river drainage system, local factors at PA level (presence of game reserves and land use/encroachment). There was no significant association between tsetse density and prevalence of trypanosomes in cattle. Total tsetse saliva and recombinant TsalI protein coated indirect ELISA are sensitive immunological probes to detect contact with tsetse flies. The new serological test at hand is a sensitive indicator that can differentiate regimens of tsetse fly bites (various degree of exposure of animals) and that can also detect tsetse fly bite after a prolonged period without exposure. The overall prevalence of trypanosome infection in tsetse fly populations was 10.3\% and 31.0\% for the field survey data and laboratory experiment data, respectively. Both spatial (country) and temporal (year of publication) variation are noticed in trypanosome infection prevalence of field collected tsetse flies. The lack of association between tsetse density and prevalence of trypanosomes in cattle needs further investigation on the role of Tabanus and Stomoxys for mechanical trypanosome transmission. Due to the ongoing interventions, the vector could decline to a low level beyond the fly catching capacity by traps. The current serological ELISA test could be an important surveillance tool with sensitive diagnostic value.
\end{abstract}

Key words: cattle trypanosomosis, tsetsefly abundance, tsetse infections, tsetse monitoring tools 


\section{Introduction}

Tsetse fly-transmitted African trypanosomosis is caused by three groups of trypanosome species, namely congolense, vivax and brucei groups (Adams et al., 2010). The distribution of the 3 I species and subspecies of tsetse flies in 38 African countries (Jordan, I993) could be summarized into three distinct habitats, namely, (i) savannah woodlands, (ii) the watercourses and drainage systems, and (iii) dense-forests (Vale et al., 2015). In these habitats a diverse species of mammals (domestic and wild), birds and reptiles provide blood meal source for tsetse species as per tsetse host preference (Weitz, 1963; Clausen et al., 1998), thus trypanosomes have exploited this tsetse-host relationship to infect a diversity of host species (Molyneux I975; Mulla and Rickman, I988). Such infections lead to varying degrees of parasitemia, anemia, tissue damage and possibly death of the infected host depending on the trypanosome species (Mbaya et al., 2012). Trypanosomosis has a significant impact on the economy and food production in Africa (Kristjanson et al., 1999). Control interventions targeting the vector and/or the parasite are crucial in eradicating or decreasing the incidence of this devastating disease, thus, have a marked impact on the economy and food production in the region. The current trypanosomosis control options have shifted to tsetse fly control as drugs and vaccine development against the parasite have failed due to the emergence of drug resistance (Delespaux et al., 2007) and immune evasion of the parasite (La Greca and Magez, 20II), respectively. A sound understanding of the tsetse population structure, tsetse behavior, host preference of tsetse for blood meal source, tsetse habitat, distribution and abundance and its temporo-spatial heterogeneity is essential in optimising vector control strategies (Leak, I999). The last countrywide survey of tsetse fly and trypanosomosis abundance in Ethiopia dates back to the late I970s (Langridge I976). Since then, trypanosomosis interventions, environmental changes and encroachment of people have been ongoing which influence the interactions between the host, parasite and vector (Van den Bossche et al., 2010). However, tsetse fly and trypanosomosis abundance is not updated in Ethiopia. The available information is restricted to particular locations to demarcate the distribution of tsetse flies by altitude (Tikubet and Gemetchu, I984) and to study invasions of tsetse in areas where tsetse was absent before (Leak et al., I993). Information on vector and parasite distribution as well as on risk factors for their abundance are of paramount importance to control the vector and the disease with the ultimate goal of achieving elimination.

Due to the ongoing interventions, both the disease and the vector could decline to a low level beyond the diagnostic capacity of microscopy and fly catching by traps. Therefore, tools that can quantify the level of exposure of cattle to tsetse earlier are essential to set up a more reliable early warning system. Another important aspect is prevalence of trypanosome in tsetse fly population. The proportion of tsetse flies infected with trypanosomes is a function of the infection prevalence and parasitemia in the hosts. Conversely, trypanosome infection prevalence in the tsetse fly is one of the fundamental parameters of African animal trypanosomosis risk analysis in estimating the risk of an individual animal to acquire a trypanosome infection. However, the prevalence of trypanosome infections in the tsetse flies is often a neglected parameter probably due to the intensive labor required for its evaluation. 
The main objective of this $\mathrm{PhD}$ thesis was to quantify the abundance of tsetse flies using traditional traps and using anti-tsetse saliva ELISA test and the trypanosome infection prevalence in the tsetse flies and cattle with a case study in Ethiopia.

\section{Methodology}

An area-wide tsetse and cattle trypanosomosis survey was conducted in Ethiopia. Trypanosomosis survey was conducted in four Regional states (Amhara, Benishangul Gumuz, Gambella, Oromia) from 2009 to 2012 around four major river systems (Abay, Abay-Didessa, Boro-Akobo, Ghibe). Cattle of both sexes and age groups $>4,>2$ to $\leq 4$ and $\leq 2$ years were sampled. Blood samples were collected from an overall of 702I animals and examined for trypanosomes using buffy coat. Tsetse fly was sampled using I046 mono-pyramidal traps deployed in Io districts within three regional states (Amhara, Benishangul Gumuz, Oromia) from 2009 to 201 I around three major river systems (Abay, Abay-Didessa, Boro-Akobo). The species and sex of the captured tsetse flies were identified and counted. In general, cattle trypanosomosis study was conducted in 82 peasant associations (PAs) whereas a tsetse fly abundance study was conducted in 52 PAs. Of these PAs, in 30 PAs both cattle trypanosomosis and tsetse abundance study were simultaneously conducted.

The goal of this study was to develop a total saliva and rTsal ELISA test that detect host exposure assessment to tsetse bite. Mice and pigs were used for Glossina morsitans morsitans challenge in Belgium at Antwerp ITM and Gent University, respectively. Mice were divided into five groups with 8 mice in each group. Mice in group I were bitten by I fly once whereas mice in group 2 were bitten by ro flies once. Mice in group 3 were bitten by i fly 3 times per week for 3 weeks whereas those in group 4 were bitten by ro flies 3 times per week for 3 weeks. Mice in groups 5 were the negative control group. Serum sampling was conducted from all mice on 7, I4, 2I, 28, 35, 42, 56, 86, II7, 270, 390 days. Another set of experimental research was conducted on pigs using three groups. Group I had 5 pigs and each pig was bitten by 30 flies once per week for 7 weeks. Group 2 also had 5 pigs and each pig was bitten by 3 flies once per 2 weeks for 7 weeks. Groups 3 had I pig and served as the negative control group. Serum Sampling was conducted on o, I4, 2I, 28, 35, 42, 49, 56, 63, 70, 77, III days.

A systematic review was conducted based on all published information available since I950s. The set criteria for article screening revolved around trypanosome infection prevalence of tsetse flies either collected in the field or in laboratory controlled conditions. Of the 605 published studies screened, 72 studies were included in the meta-analysis whilst the remaining studies were excluded as their abstracts or their contents were unrelated to the topic.

\section{Results}

Tsetse flies and trypanosomosis are still abundant and widely distributed in Ethiopia despite decades of control efforts. Of the currently widespread distribution of four tsetse fly species (G. pallidipes, G.m. submorsitans, G. fuscipes and G. tachnoides) in Ethiopia, only 
one tsetse species has been observed in the Amhara region (G. tachnoides) and the Benishangul-Gumuz region (G. m. submorsitans) whilst more than one tsetse fly species has been observed in th Oromia region. Geographical regions with an altitude lower or equal to $1200 \mathrm{~m}$ showed the highest average count of I7.I catches/trap/day. Astonishingly, geographical regions above $1500 \mathrm{~m}$ showed an unexpected high average count of 12 . This is linked to one single PA (Abuna Gali) where the tsetse abundance was abnormally high. In almost all 52 PAs studied for tsetse abundance the number of female tsetse flies captured was higher than male flies. The overall prevalence of cattle trypanosomosis over the 82 selected PAs is 9.6\%; with o\% in $\mathrm{I} 2 \mathrm{PAs}, \mathrm{I}-5 \%$ in $\mathrm{I} 7 \mathrm{PAs}, 5-\mathrm{IO} \%$ in $28 \mathrm{PAs}, \mathrm{II}-\mathrm{I} 5 \%$ in $\mathrm{I} 5$ PAs and $15-31 \%$ in 8 PAs indicating substantial variation between the PAs. With respect to the presence of the different trypanosome species, $76 \%$ was T. congolense, $18 \% \mathrm{~T}$. vivax, $3.6 \% \mathrm{~T}$. brucei and $2.4 \%$ mixed infections of T. congolense and T. vivax. The current survey highlights regions where tsetse flies and trypanosomosis are most abundant and where frequent transmission of the trypanosomes to livestock occurs. Cattle whose residence was near major river systems had significantly higher trypanosomosis. The prevalence was $15.2 \%$ at Abay Didessa river, II. $2 \%$ at Abay, 7.I\% at Baro Akobo and 5.I\% at Ghibe river. Spatial factors such as low altitude $(<\mathrm{I} 200 \mathrm{~m})$, major drainage system, local factors at peasant association level such as the presence of game reserves particularly near Abuna Gali PA and land utilization/encroachment determine the presence of major breeding habitats for tsetse flies and thus trypanosome transmission. Surprisingly, no significant relationship (OR $=\mathrm{I}, 95 \% \mathrm{CI}$ : [0.99; $\mathrm{I}$ ], $\mathrm{p}=0.348$ ) is observed between tsetse fly density and prevalence of cattle trypanosomosis which warrants further study.

Saliva proteins of tsetse fly induce antibody production in exposed animals to tsetse bite. The induced antibodies in mice reached a peak at 28 days' post tsetse bite and its half-life decay was every 36 days. The antibodies were detectable by the current ELISA after a I-year period of non-exposure. The ELISA test detects re-exposure of mice to a single tsetse fly bite after a I-year period of non-exposure period. Both anti-saliva and anti-rTsal I IgG results in mice had above $98 \%$ intra-laboratory repeatability. Diagnostic value of the anti-saliva and rTsalı in mice was assayed by area under ROC curve (AUC) analysis and 0.94 vs. 0.82 , respectively. In ELISA of pigs, repeatability analysis of the antibody detection test in pigs indicated that both anti-saliva and rTsalı had a higher repeatability (Spearman $r=0.94$ ). Anti-tsetse saliva has a better average performance than the anti-rTsalı IgG detection test (AUC 0.96 versus 0.83 ). Boosting of 2 pigs from the low exposure group after a 2-month non-exposure period by the bites of Io flies resulted in elevated anti-saliva IgG titers but was only weakly detectable using the rTsalı-based ELISA and with higher individual variation.

Of 605 published articles, 72 published articles were selected in the systematic review. The 72 papers originated from 23 countries and overall studied on 236,740 tsetse flies. Of 72 papers 49 papers were devoted to field studies on 202,182 flies. Of the 49 papers on field studies, 39 studies used dissection on 192,338 tsetse flies to detect trypanosome in them. Whereas, of 23 papers on experimental studies using 34,558 flies, I 8 papers used the dissection method on 22,478 tsetse flies. The overall samples examined 
for trypanosome infection detection in tsetse fly were tsetse saliva spit, anal droppings (diuresis fluid), midgut, proboscis, salivary glands and/or their DNA from single fly organs, DNA and pools of DNA from whole bodies. The overall prevalence of trypanosome infection in tsetse fly populations was $10.3 \%$ and $31.0 \%$ for the field survey data and laboratory experiment data, respectively. Both spatial (country) and temporal (year of publication) variation are noticed in trypanosome infection prevalence of field collected tsetse flies. Dissection is still widely used as a diagnostic method although molecular and serological techniques are gradually finding their way as well. The sensitivity of molecular/ serological tests that were performed on positive samples (i.e. by dissection) was only around $50 \%$. The alternative diagnostic tools applied to the dissection positive samples were thus characterised by low sensitivity, and no information on specificity is available at all.

\section{Discussion}

Quantitatively, the tsetse abundance varied from PA to PA. Indeed, different ecological contexts are obviously translated into different fly densities and species compositions (Slingenbergh, I992). Low altitude was significantly and positively associated with AT. Indeed, altitude directly influences ecological parameters such as vegetation cover and structure inducing specific microclimatic zones at different elevations (Leak, I999). The tsetse fly abundance in Ethiopia is also correlated positively with the size of the drainage system. Hydrologic networks (wetlands) host the highest number of tsetse flies in Ethiopia. River size, width, depth, and flow affect the type of vegetation along river banks and the associated shade, temperature and humidity which, in turn, affect tsetse fly abundance (Bouyer et al., 2005). Cattle trypanosomosis is more abundant in lower altitudes $(<\mathrm{I} 200 \mathrm{~m})$ and larger size drainage systems which is in line with the distribution of tsetse fly abundance previously explained. The study indicated that male animals and older animals are infected more often than females and young animals. This can be explained by (i) their higher attractiveness to flies (Torr et al., 200I), (ii) the higher stress linked to their use as draft animals and (iii) the malnourishment of young males used prematurely for traction work (Simukoko et al., 2007). Surprisingly, no relationship has been found between tsetse fly abundance and trypanosomosis prevalence in this new survey. It might indicate that trypanosome transmission in the area is shifting from the tsetse fly vector to other vectors (Tabanids and Stomoxys) that can transmit the trypanosome mechanically. This is a worrisome finding and needs further investigation.

About 250 proteins are available in the saliva of G. m. morsitans (Alves-Silva et al., 20Io). Tsetse fly saliva proteins play a key role in tsetse fly feeding and trypanosome transmission but they are also highly immunogenic. Some of these saliva proteins are immunogenic (Caljon et al., 2006; Poinsignon et al., 2007 \& 2008); Caljon et al. (2006) proposed Tsal I/2 based ELISA for host exposure assessment. In this study a novel diagnostic tool was developed which is capable to assess whether and when and to what extent an individual animal has been bitten by tsetse flies. The tool is based on an indirect ELISA, and has first been successfully developed for mice and pig in this study. The 
ELISA uses either total tsetse saliva or the recombinant Tsalı protein of the tsetse fly as coating antigen. Both ELISAs provide the following information. They (i) detect the exposure of the animal to tsetse fly bites, (ii) differentiate between varying levels of tsetse fly challenge (no, low and high exposure), (iii) estimate the length of exposure to tsetse fly bites based on the persistence and dynamics of induced antibodies, (iv) detect the bite of newly arriving tsetse flies after long periods of non-exposure (tsetse fly re-invasion), (v) detect presence of any species of tsetse flies (test is not tsetse species specific). Therefore, the currently developed ELISAs are useful tools in the sero-epidemiological (prevalence) study of tsetse flies, especially for regular monitoring of tsetse fly exposure and to assess the efficiency of implemented or on-going tsetse fly control activities (Fontaine et al., 20II). This serological test could thus be used in a complementary way next to entomological surveys in tsetse fly prevalence study, or to monitor the impact of vector control programs and to detect re-invasion of areas previously cleared for tsetse flies.

Both spatial (country) and temporal (year of publication) variation are noticed in trypanosome infection prevalence of field collected tsetse flies. It is essential for targeting interventions to identify areas and time periods with high prevalence of trypanosome infection in the tsetse flies. Unfortunately, the sensitivity and specificity of currently available molecular and serological techniques work apparently suboptimal for tsetse fly samples as they are developed and optimized for trypanosome detection in the host. This warrants further investigation before they can be employed on a routine basis to determine the presence of trypanosomes in the tsetse fly. Comparing several tests on the same specimen panel would allow more accurate comparisons of the sensitivity and specificity. Integrating the prevalence of trypanosome infection in the tsetse fly in monitoring programs would allow a more precise evaluation of the risk of being infected in a particular region.

\section{Conclusion}

Tsetse flies and trypanosomes are widely distributed in Ethiopia and their abundance is affected by spatial factors such as altitude, river drainage system, local factors at PA level (presence of game reserves and land use/encroachment). Both total tsetse saliva and recombinant Tsali protein based ELISA tests (i) detect hosts that were exposed from unexposed and also (ii) sort the various level of tsetse challenges to tsetse bite. The prevalence of trypanosome infection in tsetse fly populations in the field is $10.3 \%$ and affected by spatial (country) and temporal (year) factors. Unexpectedly, the available molecular and serological techniques are not sensitive to detect effectively trypanosomes in tsetse fly and the specificity of these tests is not evaluated.

\section{Acknowledgement}

I would like to acknowledge VLIR and DGD for their financial support, Addis Ababa University for allowing study leave, and ITM for allowing access to their laboratory and providing G. m. morsitans tsetse flies. 


\section{References}

Adams, E.R., Hamilton, P.B. and Gibson, W.C., (2010). African trypanosomes: celebrating diversity. Trends in parasitology, 26(7), pp.324-328.

Alves-Silva, J., Ribeiro, J.M., Abbeele, J.V., Attardo, G., Hao, Z., Haines, L.R., Soares, M.B., Berriman, M., Aksoy, S. and Lehane, M.J., (2010). An insight into the sialome of Glossina morsitans morsitans. BMC genomics, II(I), doi I0.II86/I47I-2I64-II-2I3.

Bouyer, J., Guerrini, L., César, J., De La Rocque, S. and Cuisance, D., (2005). A phyto-sociological analysis of the distribution of riverine tsetse flies in Burkina Faso. Medical and veterinary entomology, I9(4), pp.372378.

Clausen, P. H., Adeyemi, I., Bauer, B., Breloeer, M., Salchow, F. \& Staak, C. (I998). Host preferences of tsetse (Diptera: Glossinidae) based on bloodmeal identifications. Medical and veterinary entomology, I2, pp.r69-180.

Delespaux, V. and de Koning, H.P., (2007). Drugs and drug resistance in African trypanosomiasis. Drug resistance updates, Io(I), pp.30-50.

Fontaine, A., Diouf, I., Bakkali, N., Missé, D., Pagès, F., Fusai, T., Rogier, C. and Almeras, L., (20II). Implication of haematophagous arthropod salivary proteins in host-vector interactions. Parasit \& vectors, 4 , doi I0.1186/1756-3305-4-187.

Jordan, A.M., (1993). Tsetse-flies (Glossinidae). In Medical insects and arachnids. Springer Netherlands, pp. 333-388.

Kristjanson, P.M., Swallow, B.M., Rowlands, G.J., Kruska, R.L. and De Leeuw, P.N., (I999). Measuring the costs of African animal trypanosomosis, the potential benefits of control and returns to research. Agricultural systems, 59(I), pp.79-98.

La Greca, F. and Magez, S., (20II). Vaccination against trypanosomiasis: can it be done or is the trypanosome truly the ultimate immune destroyer and escape artist? Human vaccines, 7(II), pp.I225-I233.

Langridge, W.P., (1976). A tsetse and trypanosomiasis survey of Ethiopia. Ministry of Overseas Development, UK, pp.98.

Leak, S.G., (1999). Tsetse biology and ecology: their role in the epidemiology and control of trypanosomosis. New York: CABI publishing. pp. 568.

Leak, S.G.A. and Mulatu, W., (1993). Advance of Glossina morsitans submorsitans and G. pallidipes along the Ghibe-river system in southwest Ethiopia. Acta tropica, 55(I), pp.9I-95.

Mbaya, A., Nwosu, C.O. and Kumshe, H., (2012). The mechanisms of anemia in trypanosomosis: A review. In: Dr. Donald Silverberg (Ed.) INTECH Open Access Publisher. pp.269-282.

Molyneux, D.H., (1975). Diagnostic methods in animal trypanosomiasis. Veterinary parasitology, I(I), pp.5-I7.

Mulla, A.F. and Rickman, L.R., (I988). How do African game animals control trypanosome infections? Parasitology today, 4(I2), pp.352-354.

Poinsignon, A., Cornelie, S., Remoue, F., Grébaut, P., Courtin, D., Garcia, A. and Simondon, F., (2007). Human/vector relationships during human African trypanosomiasis: initial screening of immunogenic salivary proteins of Glossina species. The American journal of tropical medicine and hygiene, 76(2), pp.327-333.

Poinsignon, A., Remoue, F., Rossignol, M., Cornelie, S., Courtin, D., Grébaut, P., Garcia, A. and Simondon, F., (2008). Human IgG antibody response to Glossina saliva: an epidemiologic marker of exposure to Glossina bites. The American journal of tropical medicine and hygiene, 78(5), pp.750-753.

Simukoko, H., Marcotty, T., Phiri, I., Vercruysse, J. and Van den Bossche, P., (2007). Heterogeneity in the trypanosomosis incidence in Zebu cattle of different ages and sex on the plateau of eastern Zambia. Acta tropica, I03(2), pp.98-ror.

Slingenbergh, J., (1992). Tsetse control and agricultural development in Ethiopia. World animal review (FAO, $70-71: 30-36$.

Tikubet, G. and Gemetchu, T., (1984). Altitudinal distribution of tsetse in the Finchaa river valley (western part of Ethiopia). Insect science and its application, 5, pp.389-395. 
Torr, S.J., Wilson, P.J., Schofield, S., Mangwiro, T.N.C., Akber, S. and White, B.N., (200I). Application of DNA markers to identify the individual-specific hosts of tsetse feeding on cattle. Medical and veterinary entomology, I5(I), pp.78-86.

Vale, G.A., Hargrove, J.W., Lehane, M.J., Solano, P. and Torr, S.J., (2015). Optimal strategies for controlling riverine tsetse flies using targets: a modelling study. PLoS neglected tropical diseases, 9(3), pp. e0003615eooo3615.

Van den Bossche, P., de La Rocque, S., Hendrickx, G. and Bouyer, J., (2010). A changing environment and the epidemiology of tsetse-transmitted livestock trypanosomiasis. Trends in parasitology, 26(5), pp.236-243.

Weitz, B., (1963). The feeding habits of Glossina. Bulletin of the world health organization, 28(5-6), p.7II. 